\section{ORIGINAL RESEARCH}

S. Coley

M. Sneade

A. Clarke

Z. Mehta

D. Kallmes

S. Cekirge

I. Saatci

D. Roy

A. Molyneux

\title{
Cerecyte Coil Trial: Procedural Safety and Clinical Outcomes in Patients with Ruptured and Unruptured Intracranial Aneurysms
}

BACKGROUND AND PURPOSE: This study arose from a need to systematically evaluate the clinical and angiographic outcomes of intracranial aneurysms treated with modified coils. We report the procedural safety and clinical outcomes in a prospective randomized controlled trial of endovascular coiling for ruptured and unruptured intracranial aneurysms, comparing polymer-loaded Cerecyte coils with bare platinum coils in 23 centers worldwide.

MATERIALS AND METHODS: Five hundred patients between 18 and 70 years of age with a ruptured or unruptured target aneurysm planning to undergo endovascular coiling were randomized to receive Cerecyte or bare platinum coils. Analysis was by intention to treat.

RESULTS: Two hundred forty-nine patients were allocated to Cerecyte coils and 251 to bare platinum coils. Baseline characteristics were balanced. For ruptured aneurysms, in-hospital mortality was 2/114 $(1.8 \%)$ with Cerecyte versus $0 / 119(0 \%)$ bare platinum coils. There were $8(3.4 \%)$ adverse procedural events resulting in neurological deterioration: 5/114 (4.4\%) with Cerecyte versus 3/119 (2.5\%) with bare platinum coils $(P=.22)$. The 6 -month mRS score of $\leq 2$ was not significantly different in $103 / 109$ $(94.5 \%)$ patients with Cerecyte and 110/112 (98.2\%) patients with bare platinum coils. Poor outcome (mRS score of $\geq 3$ or death) was 6/109 (5.5\%) with Cerecyte versus 2/112 (1.8\%) with bare platinum coils $(P=.070)$. For UIAs, there was no in-hospital mortality. There were $7(2.7 \%)$ adverse procedural events with neurological deterioration, 5/133 (3.8\%) with Cerecyte versus 2/131 (1.5\%) with bare platinum coils $(P=.13)$. There was a 6 -month mRS score of $\leq 2$ in $114 / 119(95.8 \%)$ patients with Cerecyte versus $123 / 123(100 \%)$ patients with bare platinum coils. There was poor outcome (mRS $\geq 3$ and 1 death) in 5/119 (4.2\%) patients with Cerecyte versus 0/123 (0\%) patients with bare platinum coils $(P=.011)$.

CONCLUSIONS: There was a statistical excess of poor outcomes in the Cerecyte arm at discharge in the ruptured aneurysm group and at 6-month follow-up in the unruptured group. Overall adverse clinical outcomes and in-hospital mortality were exceptionally low in both groups.

ABBREVIATIONS: $\mathrm{CCT}=$ Cerecyte Coil Trial; $I \mathrm{QR}=$ interquartile range; ISAT $=$ International Subarachnoid Aneurysm Trial; $\mathrm{mRS}=$ modified Rankin Scale; PGA = polyglycolic acid; RCT = randomized controlled trial; UIA = unruptured intracranial aneurysm; WFNS = World Federation of Neurosurgical Societies

S ince the publication of the results of the ISAT, ${ }^{1,2}$ endovascular coiling of ruptured cerebral aneurysms has become the treatment of choice in many countries, if the aneurysm is anatomically suitable. There has, however, been an anxiety in both the neurosurgical and interventional communities that coiling would not prove durable, particularly after the treatment of ruptured aneurysms, and that with time the clinical benefit observed may be lost with increased numbers of patients requiring further treatment of the aneurysms or rebleeding.

Received March 15, 2011; accepted after revision June 27

From the Department of Neuroradiology (S.C., Royal Hallamshire Hospital, Sheffield, UK; Oxford Neurovascular and Neuroradiology Research Unit (M.S., A.C., A.M.), Nuffield Department of Surgical Sciences, and Department of Clinical Neurology (Z.M.), University of Oxford, Oxford, UK; Department of Radiology (D.K.), St. Mary's Hospital, Mayo Clinic, Rochester, Minnesota; Department of Neuroradiology (S.C., I.S.), Hacettepe University Hospital, Ankara, Turkey; and Department of Radiology (D.R.), Notre Dame Hospital, Montreal, Ontario, Canada.

The article was authored by Stuart Coley, Mary Sneade, and Andrew Molyneux, with assistance from Alison Clarke, Ziyah Mehta for statistical analysis, and comments from the other authors.

The work was funded and sponsored by Micrus Endovascular Inc, but the trial was independently physician-run and the company did not have access to the data during collection and analysis.
There is undoubtedly a higher probability of incomplete angiographic occlusion in patients undergoing coiling compared with those undergoing surgical clipping, resulting in more patients having retreatment of the aneurysm in the former group, ${ }^{3}$ though the risk of late rebleeding is very low. ${ }^{4}$ In an attempt to optimize the angiographic occlusion of aneurysms, there have been incremental improvements in coil design, including the use of materials incorporated in or on the coil that could potentially provide an increase in the inflammatory response. These modified coils have become widely available in the past 5 years, and anecdotal reports have suggested improved angiographic outcomes. However, it was clear soon after the introduction of these devices that there was a need to assess systematically these new coils to address the

Please address correspondence to Andrew Molyneux, MD, Neurovascular Research Unit, Nuffield Department of Surgical Sciences, University of Oxford, West Wing, John Radcliffe Hospital Oxford OX3 9DU UK; e-mail: andy.molyneux@nds.ox.ac.uk

Indicates open access to non-subscribers at www.ajnr.org

Indicates article with supplemental on-line tables.

Indicates article with supplemental on-line figure.

http://dx.doi.org/10.3174/ajnr.A2836 
following questions: 1) Can modified coils improve the angiographic outcomes achievable with bare platinum coils? 2) Would the procedural or other risks associated with their use be the same as those seen with bare platinum coils? The need to answer these questions was perceived by a number of interventionists, and the best methodology to do this was by a prospective RCT, a study design that remains rare in this field but has significant advantages over registry studies or uncontrolled case series.

In 2005, with support from Micrus Endovascular (San Jose, California), funding was provided to conduct an independent physician-led RCT with independent blinded angiographic outcome assessment comparing PGA-loaded Cerecyte coils (Micrus Endovascular) with bare platinum coils. A protocol for this trial, the CCT, was adopted and subsequently approved by a UK Multicenter Research Ethics Committee, local ethics committees, and the institutional review board as appropriate at participating centers. Micrus Endovascular acted as sponsor, but the trial was investigator-initiated and run independent of the company, and the company has not had access to the data or source documents.

A total of 23 centers participated in this study from North America, Europe, and Japan. The primary objective was to determine whether Cerecyte coils improved the proportion of patients with angiographic occlusion of the aneurysm determined on follow-up angiography at 6 months. These results for that primary outcome, along with retreatment rates, will be published separately from this current report. The secondary objectives, namely the clinical outcomes and any differences between the groups in adverse events and complications, are reported here.

\section{Materials and Methods}

\section{Patients}

Patients were eligible for the trial with the following conditions: 1) They were between 18 and 70 years of age with a ruptured or unruptured intracranial aneurysm judged suitable for coil embolization; 2) the aneurysm was $<18 \mathrm{~mm}$ (the maximum size for Cerecyte coils at the outset of the trial); 3 ) the aneurysm neck was $\geq 2 \mathrm{~mm}$; ) they had a ruptured aneurysm resulting in a good clinical grade, WFNS 1 or 2, or they had a UIA with an mRS core of zero to two; 5) they were capable of providing their own consent; and 6) they were within 30 days following an SAH. Patients were not eligible if they met any of the following criteria: 1) There was lack of consent or they could not provide their own consent; 2) they were in a poor clinical grade, WFNS 3-5 following SAH, or mRS 3-5 with a UIA; 3) they were unwilling or unlikely to return for follow-up angiography; 4) the aneurysm size was $>18 \mathrm{~mm}$; and 5) there was a planned use of a stent during treatment. This last exclusion criterion was incorporated because the primary outcome was angiographic in nature and the planned use of stents in this trial was considered inappropriate, given the potential confounding effect of stents on recanalization rates.

Patient safety was paramount. Patients were treated with the standard institutional coiling techniques used at each center participating in the trial. As far as possible, the intention was to maintain the use of all Cerecyte and bare platinum coils, but it was at the discretion of the individual centers to use coils of other manufacturers if deemed clinically necessary (eg, the need for a particular coil size). The antiplatelet and anticoagulation regimens were left to individual operators as part of the clinical practice at each center.

\section{Procedures}

The randomization allocation was issued following registration via a Web-based randomization and data-collection system ${ }^{5}$ at the coordinating center in the Oxford Neurovascular and Neuroradiology Research Unit, based in the Nuffield Department of Surgical Sciences, Oxford University, Oxford, UK. Patients were randomized after imaging showed an aneurysm suitable for endovascular treatment. Operators were not blinded to treatment type. Baseline patient data were collected at registration, and allocation to Cerecyte or bare platinum coils was by block randomization stratified by a ruptured or unruptured target aneurysm, maximum aneurysm lumen size, and neck size to ensure balance between the 2 groups. All data are held independent of the company on a secure server of the Oxford University computer network.

The clinical outcome measure used was the mRS. This measure was collected as part of a self-assessed questionnaire usually completed by the patients or, in some cases, by caregivers or a research coordinator. This was done at 6 months (or at first angiographic follow-up) and again between 12 and 24 months if the patient returned for further follow-up.

An independent Trial Steering Committee was responsible for the overall conduct of the trial. Unblinded data provided by the trial statistician were reviewed at intervals by an independent Data-Monitoring Committee to ensure patient safety. The trial conformed to the UK Good Clinical Practice and Research Governance Guidelines. The trial coordinator visited participating centers to ensure compliance, collect missing data, and conduct a random audit of clinical notes and trial records to ensure accuracy of reporting.

\section{Statistical Methods}

The statistical plan was clearly laid out in the peer-reviewed protocol before commencement of the trial. A sample size of 250 in each arm of the study was calculated at a significance level of $P=.05$ and an $80 \%$ power to detect noninferiority between the groups, assuming that a rate of adverse events at 6 months was 25\% and allowing a 5\% attrition rate. Primary analyses were by intention to treat. Adverse events and neurologic status were compared with the 1-sided P value of .05 by the Blackwelder test. Other comparisons were made by using the $t$ test, Mann Whitney $U$ test, Fisher exact test, or $\chi^{2}$ test with a 2 -sided $P$ value of .05, as appropriate. During the editorial review process, we were asked to reanalyze the data, excluding those patients who did not fully comply with the treatment allocation. The subsequent re-examination of the data to include only those patients who fully complied is referred to as the "per-protocol" analysis.

\section{Results}

The baseline characteristics of the enrolled patients are shown in On-line Table 1. There were no significant differences between the groups. Of 500 patients enrolled, 3 were excluded from the analysis: 2 because of missing consent forms on audit and 1 for exceeding the age limit. These 3 patients are known to be alive and independent. Therefore, data of 497 patients were available for analysis, 247 in the Cerecyte group and 250 in the bare platinum group (On-line Figure). 


\begin{tabular}{|c|c|c|c|c|c|c|c|c|c|}
\hline & \multicolumn{3}{|c|}{ Ruptured } & \multicolumn{3}{|c|}{ Unruptured } & \multicolumn{3}{|c|}{ All } \\
\hline & $\begin{array}{l}\text { Cerecyte } \\
(n=109) \\
(\text { No.) (\%) }\end{array}$ & $\begin{array}{c}\text { Bare } \\
\text { Platinum } \\
(n=112) \\
(\text { No.) }(\%)\end{array}$ & $P$ & $\begin{array}{l}\text { Cerecyte } \\
(n=119) \\
(\text { No.) }(\%)\end{array}$ & $\begin{array}{c}\text { Bare } \\
\text { Platinum } \\
(n=123) \\
(\text { No.) }(\%)\end{array}$ & $P$ & $\begin{array}{c}\text { Cerecyte } \\
(n=228) \\
(\text { No.) }(\%)\end{array}$ & $\begin{array}{c}\text { Bare } \\
\text { Platinum } \\
(n=235) \\
(\text { No.) }(\%)\end{array}$ & $P$ \\
\hline$\overline{\mathrm{mRS}} 0$ & $64(58.7)$ & $62(55.4)^{\mathrm{a}}$ & & $93(78.2)^{\mathrm{a}}$ & $100(81.3)^{\mathrm{a}}$ & & $157(68.9)$ & $162(68.9)$ & \\
\hline mRS 1 & $31(28.4)^{\mathrm{b}}$ & $43(38.4)^{b}$ & & $17(14.3)$ & $16(13.0)$ & & $48(21.1)$ & $59(25.1)$ & \\
\hline mRS 2 & $8(7.3)$ & $5(4.5)$ & & $4(3.4)$ & $7(5.7)$ & & $12(5.3)$ & $12(5.1)$ & \\
\hline mRS 3 & $2(1.8)$ & $0(0.0)$ & & $3(2.5)$ & $0(0.0)$ & & $5(2.2)$ & $0(0.0)$ & \\
\hline mRS 4 & $1(0.9)$ & $1(0.9)$ & & $1(0.8)$ & $0(0.0)$ & & $2(0.9)$ & $1(0.4)$ & \\
\hline mRS 5 & $0(0.0)$ & $0(0.0)$ & & $0(0.0)$ & $0(0.0)$ & & $0(0.0)$ & $0(0.0)$ & \\
\hline Death $^{\mathrm{c}}$ & $3(2.8)$ & $1(0.9)$ & & $1(0.8)$ & $0(0.0)$ & & $4(1.8)$ & $1(0.4)$ & \\
\hline$m R S \geq 3$, including death ${ }^{d}$ & $6(5.5)$ & $2(1.8)$ & .070 & $5(4.2)$ & $0(0.0)$ & 0.011 & $11(4.8)$ & $2(0.9)$ & .0049 \\
\hline
\end{tabular}

${ }^{a}$ Four patients with mRS 0 had first follow-up at $>12$ months (14, 20, 21, and 22 months).

b Two patients with mRS 1 had first follow-up at $>12$ months (13 and 14 months).

${ }^{c}$ Causes of death: in-hospital: 1 delayed cerebral ischemia due to vasospasm, 1 postprocedural acute rebleed. Postdischarge: 1 pulmonary embolism, 1 coronary disease, and 1 respiratory.

$\mathrm{d} P$ values are for 1 -sided Blackwelder tests.

Compliance with Coil Allocation and Technical Success

There was a high level of compliance with the treatment allocations. Two hundred twenty-two of 247 patients randomized to Cerecyte received their allocation at the first procedure; $3 / 247$ (1.2\%) crossed over to receive bare platinum coils only, and $15 / 247(6.1 \%)$ received a mixture of Cerecyte or bare platinum coils or coils of other manufacturers. Seven of 247 cases $(2.8 \%)$ were endovascular treatment failures in which no coils were placed at the first procedure. One patient of this 7 with a UIA in the Cerecyte group was successfully treated 1 week later.

Two hundred thirty-three of 250 patients randomized to platinum received their allocation, 3/250 (1.2\%) received $\mathrm{Mi}$ crus bare platinum and Cerecyte coils, and 7/250 (2.8\%) received a mixture of Micrus and bare platinum coils of other manufacturers. There were $7 / 250(2.8 \%)$ endovascular treatment failures at the first procedure.

In 3 cases with a UIA, a stent was deployed during the procedure as a "rescue measure." These patients are included in the clinical outcomes but would be excluded from the angiographic analysis as outlined in the protocol. A per-protocol analysis was also conducted on the basis of the type of coil actually placed.

\section{Six-Month (or First Follow-Up) Clinical Outcomes}

Clinical outcome was assessed by mRS based on the intentionto-treat analysis as shown in Table 1 .

\section{Ruptured Aneurysms}

In the ruptured aneurysm group, there was no significant difference in the clinical outcomes between the Cerecyte and bare platinum allocations; 213/221 (96.4\%) patients were independent at 6 months ( $\mathrm{mRS}$ score of $0-2$ ).

There were $6 / 109(5.5 \%)$ and $2 / 112(1.8 \%)$ poor patient outcome (mRS 3-5) in the Cerecyte and bare platinum allocations, respectively. This was not statistically significant $(P=$ .07). On a per-protocol analysis, 2 patients in the Cerecyte allocation who did not receive Cerecyte coils were mRS 2 or worse at 6 months. This changed the $P$ value slightly on the 6-month clinical outcomes $(P=.19)$.

A total of 4 patients died, 2 in-hospital, 1 from delayed cerebral ischemia due to vasospasm, and one due to an acute rebleed postprocedure. There were 2 late deaths after discharge, 1 due to respiratory obstruction and cardiac arrest and a second due to coronary disease.

\section{UIAs}

In the UIA group, 237/242 (97.8\%) patients were independent at 6 months (mRS $0-2$ ). In the Cerecyte allocation, there was a small excess of poor outcomes (mRS 3 or worse), including 1 death postdischarge due to a pulmonary embolus, 5/119 $(4.2 \%)$ compared with the bare platinum group 0/123 (0\%), which reached statistical significance $(P=.011)$. The 3 patients in the Cerecyte allocation who were self-assessed as mRS 3 at 6-month follow-up were mRS 0 or 1 at discharge. This difference did not change on the per-protocol analysis.

\section{Use of Assist Devices}

Balloon-assist usage was reported in 99/233 (42.5\%) patients with ruptured aneurysms and 126/264 (47.7\%) of patients with UIAs. These rates did not differ between the groups, 113/ $247(45.7 \%)$ and $112 / 250(44.8 \%)(P=.86)$ in the Cerecyte and bare platinum groups, respectively.

\section{Adverse Procedural Events and Clinical Outcomes}

A total of 497 first procedures were reported, of which 483 (97.2\%) were successful. Adverse procedural events are shown in On-line Table 2, and adverse periprocedural events leading to neurological deterioration in On-line Table 3.

In the patients with ruptured aneurysms, the procedural risk of aneurysm rupture was $13 / 233$ (5.6\%), overall, $8 / 114$ $(7.0 \%)$ and 5/119 (4.2\%) in the Cerecyte and bare platinum groups, respectively. These rates did not differ significantly between the 2 allocated groups $(P=.18)$. Neurologic deterioration was noted in 3 of these 13 patients (On-line Table 3 ). The per-protocol analysis reduced the number of procedural ruptures in the Cerecyte allocation by 3 (5/108, 4.6\%). The overall observed risk of any thromboembolic complication detected during or immediately after the procedure, with or without neurologic deterioration, was 11/233 (4.7\%); 7/114 $(6.1 \%)$ and $4 / 119(3.4 \%)$ in the Cerecyte and bare platinum groups, respectively $(P=.16)$.

In the patients with a UIA, the risk of procedural rupture was $5 / 264(1.9 \%)$ overall; $3 / 133(2.3 \%)$, and $2 / 131(1.5 \%)$ in 


\begin{tabular}{lccc}
\hline $\begin{array}{l}\text { Table 2: Discharge outcome, destination, and length of stay for } \\
\text { ruptured aneurysms }\end{array}$ & & & \\
\hline & & Bare & \\
& Cerecyte & Platinum & \\
& $(n=114)$ & $(n=119)$ & \\
Neurological Status at Discharge & $($ No.) $(\%)$ & $($ No.) $(\%)$ & $P$ \\
\hline WFNS 1 & $98(86.0)$ & $102(85.7)$ & \\
WFNS 2 & $10(8.8)$ & $16(13.4)$ & \\
WFNS 3 & $1(0.9)$ & $1(0.8)$ & \\
WFNS 4 & $3(2.6)$ & $0(0.0)$ & \\
Death & $2(1.8)$ & $0(0.0)$ & \\
WFNS $\geq 3$, including death & $6(5.3)$ & $1(0.8)$ & .025 \\
Destination at discharge & & & \\
Home & $83(72.8)$ & $94(79.0)$ & \\
Other hospital & $26(22.8)$ & $23(19.3)$ & \\
Rehabilitation & $3(2.6)$ & $2(1.7)$ & \\
Death & $2(1.8)$ & $0(0.0)$ & \\
Home vs rest & $83(72.8)$ & $94(79.0)$ & .13 \\
Length of stay ${ }^{b}$ (median) (IOR) & $6(3-11)$ & $7(3-11)$ & .54 \\
\hline
\end{tabular}

a $P$ values for 1 -sided Blackwelder tests unless stated otherwise.

b $P$ values for Mann-Whitney $U$ tests.

the Cerecyte and bare platinum groups, respectively. The rates were not significantly different between treatment groups $(P=.33)$. The observed risk of any thromboembolic complication, which may or may not have resulted in neurologic deterioration, detected during or immediately after the procedure was 17/264 (6.4\%) overall and 10/133 (7.5\%) and 7/131 $(5.3 \%)$ in the Cerecyte and bare platinum groups, respectively $(P=.24)$.

Other causes of reported procedural adverse events for both Cerecyte and bare platinum allocations are listed in the footnotes to On-line Table 2.

The periprocedural adverse events leading to any neurological complications (permanent or transient) are shown in On-line Table 3 . There were no significant differences between groups in the frequency of adverse events leading to neurologic deterioration, 10/247 (4.0\%) for Cerecyte and 5/250 $(2.0 \%)$ for bare platinum coils $(P=.091)$. Neurological deterioration due to any cause was observed in $8 / 233(3.4 \%)$ patients in the ruptured group and 7/264 (2.7\%) in the UIA group.

IV antiplatelet drugs (abciximab [ReoPro] or eptifibatide [Integrilin]) were used in 5 patients with a ruptured aneurysm and in 12 patients with a UIA.

On-line Table 4 lists all reported postprocedural adverse events, both neurologic and non-neurologic, occurring $>24$ hours after the procedure and before discharge. This Table reports by events rather than patients because some patients experienced $>1$ event. In patients with ruptured aneurysms, delayed cerebral ischemia due to vasospasm was reported in 29/233 (12.4\%); 14 in the Cerecyte and 15 in the bare platinum groups. It was also reported in 1 patient during treatment of a UIA. This patient had presented with a third-nerve palsy and had a procedural rupture during induction of anesthesia with subsequent SAH. There was no significant difference between the allocated groups in the frequency or pattern of postprocedural adverse events. Hydrocephalus, either acute or delayed, was only observed in patients who sustained an SAH. It was not observed at any time without an SAH.

Clinical outcome at discharge, destination at discharge, and length of stay for patients are shown in Tables 2 and 3.

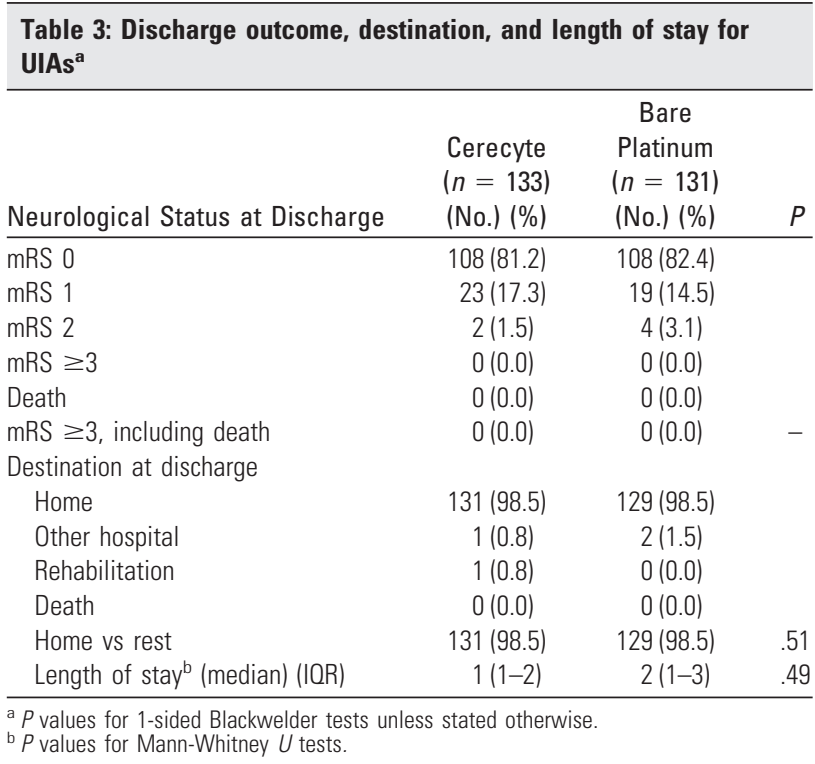

For those with ruptured aneurysms, in-hospital mortality occurred in $2 / 233(0.9 \%)$ patients. One patient died of an acute rebleed on the day of the coiling, and 1 died due to delayed cerebral ischemia due to vasospasm. There were 200/233 (85.8\%) patients who were fully alert and neurologically intact (WFNS grade 1) at discharge from the neurosurgical unit, and 26/233 (11.2\%) were graded WFNS 2 at discharge. One hundred seventy-seven of $233(76.0 \%)$ patients were discharged to home, $49 / 233(21.0 \%)$ to the referring hospital, and 5/233 (2.1\%) to a rehabilitation unit. The median length of stay was 6 and 7 days for Cerecyte and bare platinum allocations, respectively, with an IQR range of 3-11 days for both groups $(P=.54)$. Poor neurologic status at discharge (WFNS $\geq 3$ ) was significantly worse in the Cerecyte group, 6/114 (5.3\%), compared with the bare platinum group, $1 / 119(0.8 \%)(P=.025)$. When a perprotocol analysis of discharge outcomes was conducted, this difference disappeared ( $3 / 108$ and $1 / 115$ respectively) and the $P$ value was not significant $(P=.14)$.

For those patients treated for a UIA, 258/264 (97.7\%) were mRS 0 or 1 and 6/264 (2.3\%) were mRS 2 at discharge. There were no in-hospital deaths, and no patient was graded $m R S \geq 3$ at discharge. Two hundred sixty of 264 (98.5\%) patients were discharged to home, $3 / 264(1.1 \%)$ to another hospital, and 1 to a rehabilitation unit. The median length of stay was 1 (IQR 1-2) day in the Cerecyte allocation and 2 (IQR $1-3)$ in the bare platinum allocation $(P=.42)$. There was no difference to this result on the per-protocol analysis.

\section{Discussion}

Small single-center case series suggest that PGA-loaded Cerecyte coils are safe, ${ }^{6-13}$ and there is evidence that these coils are associated with less aneurysm recurrence in some matched case series. ${ }^{7,13}$ However, compelling evidence-based data for either of these observations is limited. The purpose of the CCT was to test formally the efficacy and safety of Cerecyte coils against bare platinum coils in the context of an RCT, and this article documents the adverse procedural events and clinical outcome data, both secondary end points of the trial. The 
results presented in this article provide additional evidence for the safety of both types of coil used in the treatment of ruptured and unruptured cerebral aneurysms. The use of Cerecyte coils was not associated with an increased incidence of aneurysm perforation, thromboembolism, neurologic deterioration, delayed cerebral ischemic deficit, or hydrocephalus. The risk of procedural rupture was highest among the patients with SAH (5.6\%), and the observed incidence of this event is in line with previous reports. ${ }^{14,15}$ Fewer procedural ruptures were observed in the UIA group (1.9\%) than in the ruptured group; this group included 2 patients with acute oculomotor palsies at presentation, of whom 1 had aneurysm rupture during anesthesia induction. Only 5 of the 18 patients experiencing a procedural rupture in the CCT deteriorated neurologically. It is possible that there was a greater tendency to report small subclinical perforations in the setting of a clinical trial, or perhaps the increased use of balloons, not only for remodeling but for emergency temporary vessel occlusion, may have limited the morbidity from this complication.

Only 5 of the 28 reported thromboembolic events in the entire cohort were associated with neurological decline, suggesting that this complication was either clinically silent, effectively managed, or reported when small nonocclusive clots observed on angiography had developed at the aneurysm neck.

A similar proportion of patients in each arm could not be treated by endovascular means $(2.8 \%)$, and failed attempts were largely attributed to anatomic factors rather than to primary coil failure. Our findings indicate that operators were able to safely deploy both coil types, which is consistent with anecdotal experience indicating that the Cerecyte coils are similar in performance to their bare platinum counterparts. While operators were not blinded to the coil type for practical reasons, there was no indication from the investigators that they could detect any difference in the performance of the 2 coil types.

The CCT shows a nonsignificant trend toward more adverse procedural events in the ruptured aneurysms treated with Cerecyte coils $(P=.052)$, but the fact that this difference was not present on the per-protocol analysis suggests that this may have been a chance effect $(P=.28)$.

There was no difference between the per-protocol and intention-to-treat analyses within the UIA group, and these include non-neurological complications and those complications, such as guide-catheter dissection, that cannot be directly attributed to a specific coil type. Such figures may appear somewhat high compared with those presented in certain case series $^{14-19}$; however, this could be explained by the completeness and range of the data recorded.

Comparing the reported adverse procedural complication/ event rates from different case series, registries and RCTs may be of limited value. Almost inevitably there are substantial differences between studies in terms of methodology, clinical definitions, completeness of data capture, and presentation of results. Reporting of non-neurological complications is often incomplete without prospective data collection and proscriptive pro formas, and those events that do not result in clinical deterioration may remain unreported. Further problems also arise when it is unclear whether an observed adverse clinical event can be directly attributed to a procedural complication or whether it is partly or solely related to the primary neurological insult (eg, SAH). As a consequence, it is more valuable to compare different patient groups within the same study/ trial, than try to draw comparisons with historical series.

In patients undergoing treatment of a UIA, hydrocephalus would not be expected to occur. The issue of delayed hydrocephalus in UIAs treated with other modified coils has been reported..$^{20}$ In the CCT, there was no difference in the frequency of reported hydrocephalus between the groups. The 2 cases of hydrocephalus that were reported in the CCT in the UIA group were associated with procedural aneurysm ruptures. There were no reports of delayed hydrocephalus occurring in a UIA treated with either coil type.

Increasingly, emphasis has been placed on the use of patient-assessed clinical outcome data by using standardized scoring systems for the assessment of specific cerebrovascular interventions, either as a comparison between 2 technologies (eg, coils/clips, different coil types) or against the natural history of the untreated disease (eg, IV thrombolysis for acute ischemic stroke). While comparison of procedural complication rates remains complex and often unrewarding as an indicator of the efficacy of a given intervention, the use of an outcome scale, such as the mRS score, allows meaningful comparison to be made with previous reports, assuming the data are complete and there are close similarities in patient demographics.

When data from both coil groups are pooled, 96.3\% of patients with SAH treated in the CCT achieved independent living by 6 months ( $m R S 0-2$ ), with a $1.8 \%$ mortality. A small excess of poor outcomes $(\mathrm{mRS} \geq 3)$ at initial discharge and 6-month follow-up was observed in the ruptured Cerecyte group compared with the bare platinum group. The 2 in-patient deaths were observed in the SAH group treated with Cerecyte, but neither could be directly attributed to coil factors. During the review process, we were asked to investigate the effect of a per-protocol analysis, only including patients fully complying with the allocation. This had the effect of losing statistical significance in respect to any difference in the discharge outcomes between the patients with $\mathrm{SAH}$ in the 2 groups and reducing the nonsignificant difference at 6 months in the patients with UIAs.

The clinical outcome following the treatment of UIAs in the CCT is very good. There was no in-hospital mortality in the unruptured groups, and $97.9 \%$ of patients had self-assessed their outcome as mRS $0-2$ at 6 months. There was an excess of poor outcomes observed in the UIAs treated with Cerecyte coils ( 5 versus 0 ); these included a fatal pulmonary embolus and a late ischemic stroke, both of which seem unlikely to be coil-related. This did not change on a per-protocol analysis. The data from the CCT also provide high-quality evidence of a significant improvement in patient outcome since the publication of the ISAT. There are certain similarities between the ruptured aneurysms treated in the ISAT and CCT, in that the former was also a trial that consisted largely of patients with good-grade SAH (89\% WFNS 1 and 2) with small anterior circulation aneurysms (only $8 \%$ were $>11$ $\mathrm{mm}$ ). ISAT reported a 1 -year mortality rate of $8.0 \%$, with $76.5 \%$ of patients with coiling having an mRS of $0-2$ (CCT, $1.8 \%$ and $96.3 \%$, respectively).

CCT had some limitations. First, operators were not 
blinded to coil type, and thus bias towards 1 coil or the other might have introduced some bias regarding treatment behavior during the procedure or reporting of events. Second, the small size of the study and the low event rate in respect to adverse clinical outcomes make any direct comparison between the 2 devices in respect to safety problematic, and these features mean that any subgroup analyses would not be meaningful. However, these were secondary objectives of the trial, and the primary outcome, in respect to the core lab analysis (Dr Allan Fox, Sunnybrook Health Sciences Centre, Toronto, Canada) of angiographic results, will, we hope, in the near future provide an answer to the question of whether Cerecyte coils improve angiographic occlusion.

\section{Conclusions}

The observed clinical outcomes in this study show no significant differences in the adverse procedural events or procedural neurologic complications between the Cerecyte and bare platinum coils. At 6-month follow-up in the unruptured group, there was an excess of patients with mRS 3 or worse in the Cerecyte arm, but the number of events was small ${ }^{5}$ and some of the events are unlikely to have been related to the coil type. The overall clinical outcomes for both ruptured and unruptured aneurysm treatments are better than any previously reported in a prospective randomized trial, with $96.3 \%$ of patients treated after SAH independent at 6 months and $97.9 \%$ of patients, after coiling of UIAs.

\section{Acknowledgments}

We are grateful to all the investigators and coordinators for the support and hard work in enrolling patients, completing data collection forms, and participating in the study.

\section{CCT Participating Centers and Investigators}

The CCT participating centers are listed in center order, with total patients enrolled in each center in parentheses, and the list includes the principal investigators and trial coordinators. Center 01: Royal Hallamshire Hospital, Sheffield, UK (53), Stuart Coley and Tim Hodgson, Sally Fleming and Bridget Billingham; Center 02: Wessex Neurological Center, Southampton, UK (4), John Millar, Sarah Halcrow; Center 03: Massachusetts General Hospital, Boston, Massachusetts (17), Johnny Pryor, Teresa VanderBoom and Judith Oakley; Center 04: King's College Hospital, London, UK (17), Neil Deasy and Tim Hampton, Liane Cook; Center 05: Hacettepe University Hospital, Ankara, Turkey (122), Saruhan Cekirge and Isil Saatci, Onur Ergun and Serdar Geyik; Center 06: Southern General Hospital, Glasgow, UK (28), Sarah Jenkins, Irene Scott; Center 07: St. Mary's Hospital, Mayo Clinic, Rochester, Minnesota (43), Dave Kallmes, Leigh Gray; Center 08: Institute of Neuroradiology, University of Frankfurt, Germany (9), Richard du Mesnil de Rochemont; Center 09: Newcastle General Hospital, Newcastle Upon Tyne, UK (16), Anil Gholkar, Nicola Hind; Center 10: St. Luke's Episcopal Hospital, Houston, Texas (3), Michel Mawad, Sheila Moore; Center 11: John Radcliffe Hospital, Oxford, UK (9), James Byrne, Alison Clarke; Center 12: Montreal University Hospital Center, Notre Dame Hospital, Montreal, Quebec, Canada (100), Jean Raymond, Daniel Roy, Alain Weill and François Guilbert,
Vicky Thiffault, Marcia Loor, Guylaine Gevry and Suzanne Nolet; Center 13: University Hospital, Hamburg, Germany (11), Oliver Wittkugel, Cornelia Thieme; Center 15: Stanford University Medical Center, Stanford, California (1), Michael Marks, Mary Marcellus; Center 16: Saarlandes University Hospital, Homburg, Germany (3), Iris Grunwald; Center 17: Barrow Neurological Institute, Phoenix, Arizona (4), Felipe Albuquerque, Mary Harrigan and Cheryl Kelly; Center 18: Western General Hospital, Edinburgh, UK (12), Phil White, Lynn Forrester; Center 19: University of Wisconsin, Madison, Wisconsin (3), David Niemann and Beverly Aagaard Kienitz, Kari Pulfer and Julie Grinde; Center 20: Kobe City General Hospital, Kobe, Japan (15), Nobuyuki Sakai; Center 21: Oregon Health and Science University, Portland, Oregon (19), Stan Barnwell and Wayne Clark, Sarah Jamieson; Center 22: Stony Brook University Medical Center, Stony Brook, New York, (5); Henry Woo, Dawn Madigan, Marlene Baumeister and Susan Fiore; Center 23: University of Virginia, Charlottesville, Virginia (4), Avery Evans, Patty Schweickert and Claire McKinley; Center 24: University Hospital of Wales, Cardiff, UK (2), Raed Alkilani.

\section{CCT Steering Committee}

Richard Kerr (Chair) Department of Neurosurgery, John Radcliffe Hospital, Oxford, UK; Nick Higgins, Department of Neuroradiology, Addenbrooke's Hospital, Cambridge, UK; Dennis Briley, Department of Neurology, John Radcliffe Hospital, Oxford, UK; Ziyah Mehta (statistician), Department of Clinical Neurology, University of Oxford, UK; Julia Yarnold, Oxford Neurovascular and Neuroradiology Research Unit, Nuffield Department of Surgical Sciences, University of Oxford, UK; Andrew Molyneux (Chief Investigator), Oxford Neurovascular and Neuroradiology Research Unit, Nuffield Department of Surgical Sciences, University of Oxford, UK.

\section{Data-Monitoring Committee}

Peter Rothwell (Chair), Department of Clinical Neurology, University of Oxford, UK; David Mendelow, Department of Neurosurgery, Newcastle General Hospital, Newcastle, UK; Martin Jeffree, Department of Neuroradiology, Hurstwood Park, Haywards Heath, UK.

Disclosures: Stuart Coley—RELATED: Consulting Fee or Honorarium: Micrus, ${ }^{*}$ Details: An honorarium was paid to each recruiting center for each randomized patient in the CCT, Support for Travel to Meetings for the Study or Other Purposes: Micrus, ${ }^{*}$ Details: Stuart Coley has accepted travel expenses/accommodations from Micrus to present interim data on the CCT at Val D'Isere France in 2008 and 2009; expenses paid for attendance/ presentation at Cerecyte investigators meeting in Boston 2010. Mary Sneade-RELATED: Support for Travel to Meetings for the Study or Other Purposes: Micrus, ${ }^{*}$ Comments: travel for site visits as part of study protocol and meetings for study setup and data presentation. David Kallmes—RELATED: Grant. Micrus, ${ }^{*}$ Comments: research support for a clinical trial; UNRELATED: Grants/Grants Pending: ev3, ${ }^{*}$ NFocus, ${ }^{*}$ Sequent, ${ }^{*}$ MicroVention, ${ }^{*}$ Cook, ${ }^{*}$ ArthroCare, ${ }^{*}$ CareFusion, ${ }^{*}$ Payment for Development of Educational Presentations: CareFusion, ${ }^{*}$ ev3;* Travel/Accommodations/Meeting Expenses Unrelated to Activities Listed: MicroVention.* Andrew Molyneux — RELATED: Consulting Fee or Honorarium: Micrus, Comments: financial support for the trial including salary and research cost to the Oxford Radcliffe Hospitals NHS Trust; Support for Travel to Meetings for the Study or Other Purposes: Micrus, Comments: investigator meeting support; UNRELATED: Board Membership: Micrus, Comments: advisory board member; Speaker Bureau: Micrus, Details: presentations at scientific meetings including the American Society of Neuroradiology for hotel and travel; Consultant. Micrus, Details: consulting agreement for trial development and research and development; Expert Testimony. UK courts. Daniel Roy-RELATED: Grant. Micrus, ${ }^{*}$ Comments: payments for research coordinator based on number of recruited patients; UNRELATED: Grants/Grants Pending: Terumo, Comments: grant for the PRET 
study. Saruhan Cekirge — UNRELATED: Grant/Grants Pending: Micrus. ${ }^{*}$ Isil Saatci-UNRELATED: Grant/Grants Pending: Micrus. * *Money paid to the institution.

\section{References}

1. Molyneux AJ, Kerr RSC, Stratton I, et al. International Subarachnoid Aneurysm Trial (ISAT) of neurosurgical clipping versus endovascular coiling in 2143 patients with ruptured intracranial aneurysms: a randomised trial. Lancet 2002;360:1267-74

2. Molyneux AJ, Kerr RS, Yu L-M, et al. International Subarachnoid Aneurysm Trial (ISAT) of neurosurgical clipping versus endovascular coiling in 2143 patients with ruptured intracranial aneurysms: a randomised comparison of effects on survival, dependency, seizures, rebleeding, subgroups, and aneurysm occlusion. Lancet 2005;366:809-17

3. Campi A, Ramzi N, Molyneux AJ, et al. Retreatment of ruptured cerebral aneurysms in patients randomized by coiling or clipping in the International Subarachnoid Aneurysm Trial (ISAT). Stroke 2007;38:1538-44

4. Molyneux AJ, Kerr RSC, Birks J, et al. Risk of recurrent subarachnoid haemorrhage, death or dependence and standardised mortality ratios after clipping or coiling of an intracranial aneurysm in the International Subarachnoid Aneurysm Trial (ISAT): long-term follow-up. Lancet Neurol 2009;8:427-33

5. InferMed. Macro electronic data capture. http://www.infermed.com. Accessed 31 Jan 2011

6. Linfante I, DeLeo MJ, Gounis MJ III, et al. Cerecyte versus platinum coils in the treatment of intracranial aneurysms: packing attenuation and clinical and angiographic midterm results. AJNR Am J Neuroradiol 2009;30:1496-501

7. Bendszus M, Solymosi L. Cerecyte coils in the treatment of intracranial aneurysms: a preliminary clinical study. AJNR Am J Neuroradiol 2006;27: 2053-57

8. Bendszus M, Bartsch AJ, Solymosi L. Endovascular occlusion of aneurysms using a new bioactive coil: a matched pair analysis with bare platinum coils. Stroke 2007;38:2855-57. Epub 2007 Aug 23

9. Butteriss D, Gholkar A, Mitra D, et al. Single-center experience of Cerecyte coils in the treatment of intracranial aneurysms: initial experience and early follow-up results. AJNR Am J Neuroradiol 2008;29:53-56

10. Veznedaroglu E, Koebbe CJ, Siddiqui A, et al. Initial experience with bioactive Cerecyte detachable coils: impact on reducing recurrence rates. Neurosurgery 2008;62:1516-24

11. Castro E, Villoria F, Castaño C, et al. Spanish registry for embolization of small intracranial aneurysms with Cerecyte coils (SPAREC) study: early experience and mid-term follow-up results. Interv Neuroradiol 2008;14:375-84

12. Leonardi M, Dall'olio M, Vasquez OO, et al. Preliminary experience of Cerecyte coils in the treatment of intracranial aneurysms. Interv Neuroradiol 2008 14:285-92. Epub 2008 Oct 8

13. Geyik S, Ertugral O, Yavuz K, et al. Comparison of bioactive coils and bare platinum coils for treatment of intracranial aneurysms: a matched pair analysis. J Neurosurg 2010;112:709-13

14. Ross IB, Dhillon GS. Complications of endovascular treatment of cerebral aneurysms. Surg Neurol 2005;64:12-19

15. Cloft HJ, Kallmes DF. Cerebral aneurysm perforations complicating therapy with Guglielmi detachable coils: a meta-analysis. AJNR Am J Neuroradiol 2002;23:1706-09

16. Henkes H, Fischer S, Weber W, et al. Endovascular coil occlusion of 1811 intracranial aneurysms: early angiographic and clinical results. Neurosurgery 2004;54:268-80

17. van Rooij WJ, Sluzewski M, Beute GN, et al. Procedural complications of coiling of ruptured intracranial aneurysms: incidence and risk factors in a consecutive series of 681 patients. AJNR Am J Neuroradiol 2006;27:1498-501

18. Brilstra EH, Rinkel GJ, van der Graaf Y, et al. Treatment of intracranial aneurysms by embolization with coils: a systematic review. Stroke 1999;30:470-76

19. Viñuela F, Duckwiler G, Mawad M. Guglielmi detachable coil embolization of acute intracranial aneurysm: perioperative anatomical and clinical outcome in 403 patients. J Neurosurg 1997;86:3:475-82

20. Marchan EM, Sekula RF Jr, Ku A, et al. Hydrogel coil-related delayed hydrocephalus in patients with unruptured aneurysms. J Neurosurg 2008;109:2: $186-90$ 